\title{
Internal current generation in respiration chambers
}

\author{
R. Saborowski \& F. Buchholz \\ Biologische Anstalt Helgoland, Meeresstation, D-27483 Helgoland, Germany
}

\begin{abstract}
A technical device generating a constant and directed current within a sealed respiration chamber is described. It does not involve any external pumps or tubing. This system is easy to handle, and improved the maintenance of theotactic pelagic species like the Northern krill (Meganyctiphanes norvegica, Crustacea) or small fishes (Gasterosteus aculeatus) under experimental conditions.
\end{abstract}

Key words: Respiration chamber, current, rheotactic animals, krill, fish

\section{INTRODUCTION}

The accurate determination of oxygen consumption rates in aquatic animals depends on appropriate systems which allow the organism under study to be maintained under close-to-natural conditions. Besides the choice of the measurement method (i.e. electrodes, optrodes, chemical) and control or manipulation of the experimental conditions (light, temperature, addition of chemicals), the construction of the respiration chamber has a significant influence on the course of the experiment. Bulnheim (1974) equipped a respiration chamber with a piece of gauze to meet the thigmotactic behaviour of the examined isopods and to reduce their activity. Chapelle \& Peck (1995) showed that the presence of a substratum to which species could attach allowed a more natural behaviour of Antarctic amphipods and decreased the respiration rates. Respiration measurements are commonly carried out in relatively small closed systems (bottle method). Certain applications, however, demand more sophisticated technical solutions. For example, the effect of hydrostatic pressure on the respiration rate of marine animals and tissues were studied by Gordon \& Thomas (1974) as well as Belman \& Gordon (1979). Systems for long-term monitoring of respiration rates were designed by Sutcliffe et al. (1975) and Quetin (1983). Eriksen \& Feldmeth (1967) introduced a water-current respirometer to simulate the conditions to which animals are exposed in running water (e.g. insect larvae).

It is far more complicated to establish appropriate conditions for pelagic organisms than it is for benthic organisms. This is particularly true for species which have a higher specific weight than water, e.g. large mysids and euphausiids. In standard respiration chambers, these species tend to cease swimming and often sink to the bottom, which does not reflect their natural behaviour. It is, therefore, important to encourage them to swim freely and to remain in the water column. Previous investigations on Antarctic krill made use of large respiration chambers (60 l) which were equipped with an external electric water-pump system that generated a current inside the chamber (Kils, 1981; Voss, pers. 
comm.). The krill orientated themselves against the current and swam permanently in the water column.

Large chambers, however, are awkward to handle in routine laboratory work and, particularly, when used on board research vessels. External tubing increases the susceptibility to the formation of air bubbles in the system and electric pumps may warm up the water. Accordingly, the aim of the present study was to develop a system which applies a continuous current inside a respiration chamber using the simplest methods. We also tried to avoid unnecessary external equipment to reduce the risk of technical problems during operation. As a result we introduce a respiration chamber containing an integrated current generator for the measurement of respiration rates in Northern krill and other pelagic species.

In addition to the current generator which is integrated in the respiration chamber, a self-contained turbulence generator is described which uses the same mechanical principle as the internal generator. It can be applied to induce a gentle water movement within small conventional containers used for respiration measurements.

\section{Description of the respiration chamber}

The Perspex chamber (Fig. 1) had a total volume of $1.65 \mathrm{l}$ and consisted of three functional units. The bottom was separated by a punctured plate and hosted a stir bar. This stir bar was driven by an electromagnetic stirrer on which the chamber was placed during operation. This compartment acted as the generator for the water movement. As a result of the rotation of the stirrer, the water was pushed by centrifugal force to the walls of this compartment and was directed through six pairs of obliquely orientated holes into the outer cylindrical compartment of the chamber. This compartment served as the maintenance compartment for the organisms under study. The species were allowed to move freely in the water column and could make use of the circular and, therefore, potentially 'endless' swimming arena. At the top, the maintenance compartment was connected via small holes with a tubular funnel. This funnel was again a cylindrical unit which, however, was much smaller in diameter than the maintenance compartment. It directed the water flow downwards back into the generator.

The integrative action of these three compartments generated a directed stream of water within the respiration chamber and supplied the maintenance compartment with a constant current. We used a waterproof electromagnetic stirrer (Variomag® Telemodul $40 \mathrm{~S}$ and no. 40151 compact stirring drive units, $\mathrm{H}+\mathrm{P}$ Labortechnik $\mathrm{GmbH}$, Munich, Germany). It allowed the running of all stirring drives (up to 8) with exactly the same rotation speed.

\section{Intensity of the induced current}

The intensity of the water current was regulated by the rotation speed of the magnetic stirrer between 120 and $930 \mathrm{rpm}$. The velocity of the current at a given speed of the stirrer was determined by measuring the duration of the complete circulation of floating particles in the maintenance compartment. The speed of the current was calculated as angular velocity $\left(\omega=\varphi t^{-1}=2 \pi t^{-1}\right)$ and expressed as $\left[\mathrm{s}^{-1}\right]$. The orbital velocity corresponds to $\nu=\omega r\left[\mathrm{~cm} \mathrm{~s}^{-1}\right]$. For example, assuming a maximum radius of the maintenance com- 


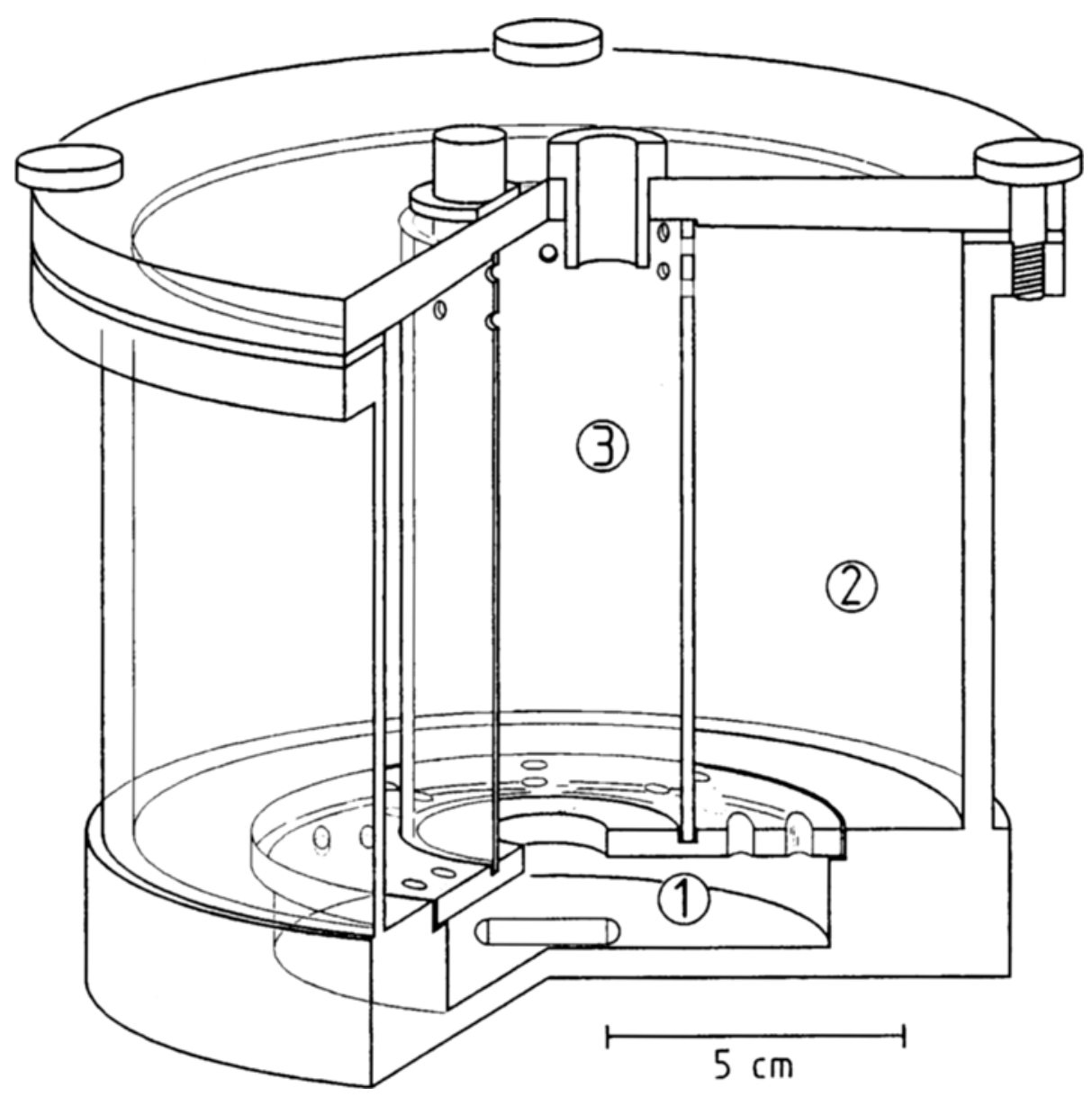

Fig. 1. Respiration chamber with an integrated, self-generating current system. The chamber consists of a generator unit (1), a maintenance compartment (2) and a funnel (3). The lid is fixed by screws and sealed by a rubber ring. The lid also hosts an electrode fitting and an extra opening for flow-through measurements or the application of test substances. Further explanations are given in the text

partment of $6.75 \mathrm{~cm}$ (circumference $42.4 \mathrm{~cm}$ ) and an angular velocity of $0.5 \mathrm{~s}^{-1}$, the orbital velocity $v$ is $3.38 \mathrm{~cm} \mathrm{~s}^{-1}$.

The relation between the rotation speed of the stirrer and velocity in the maintenance compartment followed a sigmoidal function (Fig. 2). The lowest applicable rotation speed was $130 \mathrm{rpm}$ which provided an angular velocity of $0.267 \mathrm{~s}^{-1}$. The successive increase of the rotation speed from 200 to $350 \mathrm{rpm}$ was paralleled by a continuous increase of velocity. At a rotation speed above $350 \mathrm{rpm}$ the increase in velocity ceased and the curve asymptotically converged towards a maximum. This limitation in maximal velocity is related to the construction of the specific chamber and, in particular, to the size and number of junctions between the compartments of the chamber. 


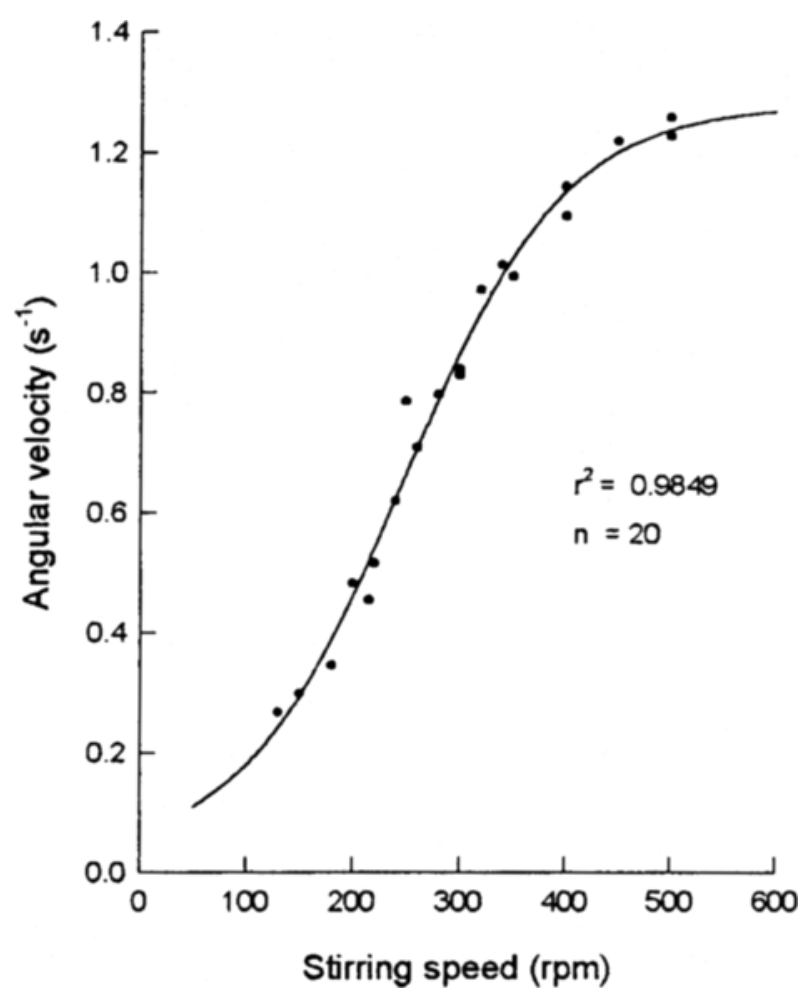

Fig. 2. Relation between stirring speed and current speed (angular velocity) within the chamber. The relation follows significantly the Boltzmann sigmoid equation $y=y_{\min }+\left(\left[y_{\max }-y_{\min }\right] /\left[1+e^{(v 50-x / s l o p e}\right]\right)$ as calculated with the computer program Prism. Graph Pad Software Inc., San Diego

\section{Turbulence generator}

The turbulence generator (Fig. 3 ) is similar to that in the system described above. The current generating unit is simplified and reduced in size for use in vials without internal stirring device. The system consists of a flat cylindrical housing (pill box shape) which contains a small magnetic stir bar. As a result of centrifugal forces an outward directed water current is induced through the lateral jets. The backstream of water is allowed through the wide opening on top of the box. This opening is covered with nylon gauze $(100 \mu \mathrm{m})$ to prevent specimens from entering the stirring compartment. This generator does not provide a directed stream of water within the respiration chamber. However, it generates a turbulence which ensures a gentle turnover of the incubation medium.

\section{Application}

The system presented here provides a simple respirometer unit that generates a permanent and constant water movement in the incubation chamber. Pelagic animals are encouraged to swim freely in the water column against a circular current. Successful 


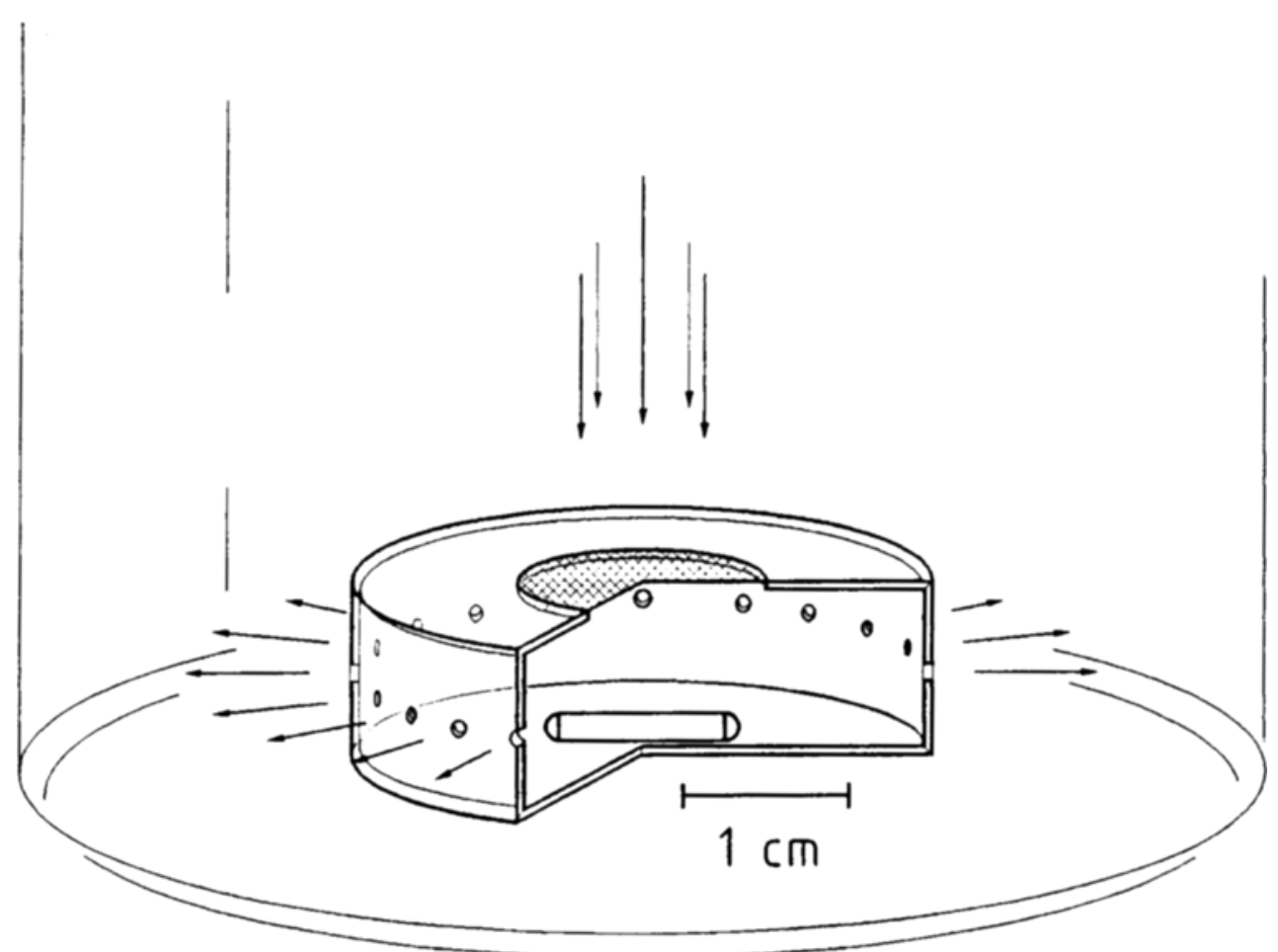

Fig. 3. Turbulence generator for use in commercial vials or bottles that are not provided with an adequate stirring device. The arrows indicate the flow of water

preliminary investigations were carried out on krill and fishes. In accordance with the dimensions of the chamber, we used animals in the size-range of $30-60 \mathrm{~mm}$.

The Northern krill, Meganyctiphanes norvegica, is a pelagic euphausiid of up to $35 \mathrm{~mm}$ body length. Since krill is not easy to maintain in the laboratory for long periods we carried out this study on board a research vessel with freshly caught animals. We introduced single animals or groups of three specimens into the chamber and observed their behaviour with a video system under infrared illumination. The animals exhibited a more active behaviour and spent more time swimming in the water column during periods of stirring. A slow and gentle current close to the lowest stirring speed was most favourable. At increased water movement the animals tended to swim more erratically and occasionally showed escape behaviour.

As an example for small fishes we studied three-spined sticklebacks (Gasterosteus aculeatus). Fish of approximately $60 \mathrm{~mm}$ length were maintained individually in the chamber. When no current was applied, the fish explored the chamber and swam freely in the maintenance compartment. As soon as the current was intensified, the fish compensated their displacement by swimming against the current. The swimming speed gradually increased as a response to the water movement. This type of chamber can easily be adapted to smaller scale applications. Of particular interest would be the 
measurement of the metabolic demand of fish larvae and juvenile fishes for studies on the expenditure of energy needed for swimming against currents.

Other features of the system are advantageous in routine application. One is the spatial separation of the electrode from the animals in the maintenance chamber. This protects the electrodes from being damaged by aggressive species. For example, some isopods and amphipods as well as grapsid decapods tend to hold on to such objects. Fish may snap at or hit the electrode. This behaviour increases the risk of damage particularly to the sensitive membranes of the electrodes. Furthermore, the current induced in the chamber causes a permanent and complete mixing of the water. This is important in avoiding partial oxygen depletion within the system. This feature of the chamber also enables the study of species which prefer shelters (e.g. juvenile lobster). The permanent current through the system supplies the electrode with a constant stream of water. This is particularly important for electrodes which consume a considerable amount of oxygen themselves.

It may be regarded as a disadvantage that the chamber consists of several parts that may increase the susceptibility of catching air bubbles during filling and closing of the chamber. However, in practice we had no problems with air bubbles, particularly when the chambers were filled and closed under water (e.g. in a bucket of appropriate size).

The turbulence generator can be applied within commercial vials or bottles which are not provided with an adequate stirring device (see also Quetin \& Mickel, 1983). It is particularly useful for experiments with small and fragile animals of a few $\mathrm{mm}$, e.g. decapod larvae or small isopods. These animals would be injured or damaged when hit by a stirrer. In our system, the stirring bar is sheltered within a housing that prevents contact with the organism but allows the flow of water through the punctured walls. Gentle turbulence is also necessary when the animals under study are too small or too inactive to produce sufficient water movement themselves for continuously exchanging the water at the tip of the electrode and, thus, ensuring a uniform oxygen distribution within the chamber. The generator can be easily driven by a magnetic stirrer which is placed below the chamber. However, when the generator is not fixed at the bottom of the chamber, a critical rotation speed of the stir bar should not be exceeded in case the entire generator starts wobbling and moving by itself.

The technical principle of the system introduced here can be easily adapted to suit various applications and is a contribution to studies on ecophysiological processes in aquatic animals under close-to-natural conditions.

Acknowledgements. We are indebted to Mr. Manfred Pieper from our institute's workshop in Hamburg for the realization of the chamber, and the crew of FS Heincke for excellent support in the field. This work was funded by the European Union within the MAST III-programme under MAS3-CT0013 (PEP).

\section{LITERATURE CITED}

Belman, B. W. \& Gordon, M. S., 1979. Comparative studies on the metabolism of shallow-water and deep-sea marine fishes. V. Effect of temperature and hydrostatic pressure on oxygen consumption in the mesopelagic zoarcid Melanostigma pammelas. - Mar. Biol. 50, 275-281.

Bulnheim, H.-P., 1974. Respiratory metabolism of Idotea balthica (Crustacea, Isopoda) in relation to environmental variables, acclimation processes and moulting. - Helgoländer wiss. Meeresunters. $26,464-480$. 
Chapelle, G. \& Peck, L.S., 1995. The influence of acclimation and substratum on the metabolism of the Antarctic amphipods Waldeckia obesa (Chevreux 1905) and Bovallia gigantea (Pfeffer 1888). - Polar Biol. 15, 225-232.

Eriksen, C. H. \& Feldmeth, C. R., 1967. A water-current respirometer. - Hydrobiologia 29, 495-504.

Gordon, M. S. \& Thomas, T. J., 1974. Comparative studies on the metabolism of shallow-water and deep-sea marine fishes. III. Apparatus for studies of tissue preparations under high hydrostatic pressures. - Mar. Biol. 28, 73-77.

Kils, U., 1981. The swimming behavior, swimming performance and energy balance of Antarctic krill, Euphausia superba. - BIOMASS Sci. Ser. 3, 1-122.

Quetin, L. B., 1983. An automated, intermittent flow respirometer for monitoring oxygen consumption and long-term activity of pelagic crustaceans. In: Polarographic oxygen sensors. Ed. by E. Gnaiger \& H. Forster. Springer, Berlin, 176-183.

Quetin, L. B. \& Mickel, T. J., 1983. Sealed respirometers for small invertebrates. In: Polarographic oxygen sensors. Ed. by E. Gnaiger \& H. Forster. Springer, Berlin, 184-189.

Sutcliffe, D. W., Carrick, T. R. \& Moore, W. H., 1975. An automated respirometer for determining oxygen uptake in crayfish (Austropotamobius pallipes [Lereboullet]) over periods of 3-4 days. - J. exp. Biol. 63, 673-688. 